Aviso importante: POLIANTEA Vol.XIII No. 24, informa que este documento es una versión anticipada en formato PDF del artículo final a publicar, que se pone a disposición del público mientras se realiza el proceso de diagramación y traducción, con el fin de anticipar la visibilidad, teniendo en cuenta el cumplimiento y aprobación del proceso editorial de la revista.

Esta versión provisional contiene el texto entregado a diagramación, la presente tiene una asignación automática de DOI que estará activa una vez sea publicada la definitiva en esta misma plataforma.

Tenga presente que esta versión puede diferir de la definitiva en pequeños detalles.

\title{
DISEÑO DE LA INTERFAZ GRAFICA DEL MÓDULO DE INFORMACIÓN INTERACTIVA PARA LA INTEGRACIÓN DE INDIVIDUOS CON CAPACIDADES DIVERSAS EN LA BIBLIOTECA PÚBLICA DEL ESTADO ZULIA.
}

\author{
Luisenia Fabiola Fernández Ochoa (Venezuela) \\ fdezl13@hotmail.com \\ Magister en Ciencias de la Comunicación \\ Universidad del Zulia
}

\author{
Derlisiret Rincón (Venenzuela) \\ derlyta@yahoo.com \\ Post-Doctor en Políticas Públicas \\ Universidad del Zulia
}

Recibido: 14 de octubre de 2016

Evaluado: 16 de mayo de 2017

Aprobado: 10 de octubre de 2017

¿Como citar este artículo?

Fernandez-Ochoa, L. F. \& Rincón, D. (2017). Diseño de la interfaz gráfica del módulo de información interactiva para la integración de individuos con capacidades diversas en la Biblioteca Pública del Estado Zulia, Poliantea 13(24), pág. XX-XX. 


\section{RESUMEN}

La presente investigación busca entender las Ciudades Inteligentes como paradigmas emergentes de desarrollo sustentable y mecanismos de inclusión. Para ello, se conciben los módulos o kioskos de información como los medios para lograr la construcción de estos modelos urbanos. Para ello, se diseñó la interfaz gráfica del módulo de información interactiva para la integración de individuos con capacidades diversas en la Biblioteca Pública del estado Zulia. Sustentado en las posturas teóricas de Aguirre (2013), Thomas (2008), y Mitchell (2007). Mediante el uso de una metodología descriptiva con un diseño de campo no experimental transeccional y documental. Se concluye que, los módulos de información interactiva, no son sólo el medio para incluir el uso de las TIC, de manera "Inteligentes" para la mejora de interacción y la creación de sistemas más amigables y el fomento de una ciudad inteligente; sino que además genera inclusión en la diversidad, aprendizaje, mejoras en pro del el medio ambiente mediante el uso de las tecnologías para la gestión (inteligente), el desarrollo sostenible del entorno y la minimización de las brechas existentes.

Palabras claves: módulos de información, integración y capacidades diversas.

\footnotetext{
* Especialista en Docencia para la Educación Superior. Magister en Ciencias Comunicación. Universidad del Zulia Profesora del Departamento de Historia de la Ciudad la Arquitectura y el Diseño de la Universidad del Zulia (LUZ). Maracaibo, Venezuela. Teléfono: 58: 412.6579339.E-mail: fdezl13@ hotmail.com

** Post-Doctor en Políticas Públicas. Dra. Ciencias Gerenciales. Profesora Titular. Departamento de Ciencias Humanas. Facultad Experimental de Ciencias (FEC) de la Universidad del Zulia (LUZ). Maracaibo, Venezuela. E-mail: derlyta@yahoo,com

*** La interfaz gráfica de este trabajo se puede consultar en línea en el siguiente link:
} 


\title{
GRAPHIC DESIGN INTERFACE MODULE INTERACTIVE INFORMATION FOR INTEGRATION OF INDIVIDUALS WITH DIFFERENT CAPABILITIES IN STATE PUBLIC LIBRARY ZULIA.
}

\section{AUTHORS: FERNANDEZ, LUISENIA MSC. And RINCÓN, DERLISIRET P.HD.}

\begin{abstract}
This research seeks to understand the Smart Cities and emerging paradigms of sustainable development and inclusion mechanisms. To do this, the modules or information kiosks as the means to achieve the construction of these urban models are conceived. To do this, the GUI interactive information module for the integration of individuals with different capacities in the Public Library of Zulia state was designed. Supported by the theoretical positions of Aguirre (2013), Thomas (2008) and Mitchell (2007). Using a descriptive design methodology transeccional and no experimental documentary field. It is concluded that interactive information modules are not only the means to include the use of ICT, "smart" way to improve interaction and creating more friendly systems and the promotion of a smart city; but also it generates inclusion in diversity, learning, improvements in favor of the environment through the use of technologies (smart) management, sustainable development of the environment and minimizing gaps.
\end{abstract}

Keywords: information modules, integration and different capacities.

\footnotetext{
* Specialist Teaching for Higher Education. Magister in Communication Sciences. Zulia University Professor, Department of History of the City of Architecture and Design at the University of Zulia (LUZ). Maracaibo, Venezuela. Telephone: 58: 412.6579339.E-mail: fdezl13@ hotmail.com

** Post-Doctor in Public Policy. Dra. Management Science. Titular teacher. Department of Human Sciences. Faculty of Sciences (FEC) at the University of Zulia (LUZ). Maracaibo, Venezuela. E-mail: derlyta @ yahoo, com

$* * *$ The graphical interface of this work is available online at the following link:
} 


\section{Introducción}

El ritmo de vida en el último siglo ha provocado un vuelco de la densidad de población. Se ha emigrado del campo a las ciudades; y ésto ha llevado al crecimiento desmesurado y en ocasiones desorganizado. El proceso aún sigue en marcha, aunque dependiendo de las zonas tiene distinta intensidad y que según la ONU en el año 2050 el 70\% de las personas vivirán en zonas urbanas.

Así pues, nace un nuevo contexto; con ello nuevos entornos desencadenando la irrupción de unas ciudades distintas a las configuraciones tradicionales; ambientes donde ahora se conjugan tecnologías y ciudadanos donde se articulan en nuevos cánones de desarrollo. Las denominadas 'smart cities' o ciudades inteligentes son entonces los espacios donde se permea la transformación y se refieren a esas ciudades que con la ayuda de la tecnología crean ambientes sanos, sustentables, amigables, inclusivos que las hacen más habitables para todos donde el Internet de los Objetos, inundan las áreas urbanas del llamado área del "futuro próximo".

Se está en presencia pues del concepto de ciudad inteligente que conlleva la implicación de la tecnología para hacer más habitables las zonas urbanas y facilitar la convivencia en lugares que en un futuro estarán superpoblados. No cabe duda, que en esta transformación una de las principales herramientas para lograrla es la expansión de Internet (TICbeat, 2011).

Al respecto Ramírez (2012), plantea que las Ciudades Inteligentes surgen como una alternativa para los alcaldes y gobernadores, dado que promueve funciones y servicios tales como: administración pública, control del tráfico, prevención del crimen, prevención de incendios, salud, educación, medio ambiente y redes residenciales, combinando la infraestructura de alta tecnología y sistemas de información disponibles en cualquier parte de las zonas urbanas. 
Así mismo, pueden definirse las Smart City (en español Ciudad Inteligente) como aquellas ciudades que usan la tecnología para hacer que tanto su infraestructura como sus componentes y servicios públicos ofrecidos sean más interactivas, eficientes y los ciudadanos puedan ser más conscientes de ellos. Se dice que son metrópolis comprometidas con su entorno, tanto desde el punto de vista medioambiental como en lo relativo a los elementos culturales e históricos.

Los ejes en los que suele incidir un proyecto de "Smart City- Ciudades Inteligentes- Telepolis - MegaTelepolis" tienen que ver con la movilidad urbana, la eficiencia energética; y en general, la gestión sostenible de los recursos, la gestión de las infraestructuras de la ciudad, el gobierno participativo y la seguridad pública así como con las áreas de salud, educación y cultura (Educared Colombia, 2012).

Rubio (2011), explica que las Smart City (Ciudad Inteligente), es un concepto que se refiere al potencial número de servicios que, a través de la conectividad, hagan de las ciudades un mejor lugar para vivir. Las Smart Cities son la representación más ambiciosa del "Internet of Things" (IOT). El objetivo es aglutinar todo un ejército de sistemas inteligentes que suministren (y gestionen) información para y a través de los ciudadanos. Aunque pueda parecer un concepto futurista, a día de hoy ya se han empezado a dar algunos pasos.

El modelo de Smart City agrupa una serie de características propias, moldeadas a través de la combinación de todos los factores que influyen en el progreso de la ciudad: económico, político, social, medioambiental, de movilidad y de calidad de vida. Partiendo de ellos, se camina hacia un modelo organizativo y tecnológico propio de una ciudad moderna. No obstante, estos modelos urbanos requieren un novedoso complejo de infraestructuras que faciliten la actividad diaria de sus habitantes para lograr que el tránsito a la denominada de una Urbe Digital a Smart City. 


\section{Generalidades de las ciudades inteligentes}

Para entender un poco más sobre este concepto emergente que irrumpe en el Siglo XXI se requiere comprender que éstas ciudades se contemplan como unidades de negocio donde se aplican principios de eficiencia empresarial a las administraciones y a todos los espacios que son aludidos por una ciudad, espacios que dejan de ser físicos y se convierten en económicos.

El viario y las redes definen el territorio y el espacio se contempla como un bien público escaso. El poder de atracción de las áreas metropolitanas ya no se basa únicamente en su capacidad de oferta de trabajo y vivienda, sino que cada vez tiene mayor importancia la dotación de servicios, tanto en cantidad como en calidad, para "satisfacer al cliente". La ciudad moderna y de calidad, ha de proveer servicios y operaciones, pero quiere también hacerlo de forma eficiente (independientemente de quién sea el operador del servicio). La ciudad ha de poder internalizar estos logros en forma de ofertas económicas más competitivas de los operadores o bien recomendar a sus operadores (la interface de la calidad de vida que percibe el ciudadano) un cambio de hábitos que permita la mejora continua.

Una vez asumida cierta racionalidad y experiencia en la construcción de infraestructuras y edificios y en el urbanismo, es la eficiencia en las operaciones la que garantiza la continuidad de los servicios y permite vencer ineficiencias funcionales y descoordinaciones.

Desde esta perspectiva, en la Sociedad del Conocimiento los ciudadanos que contemplan las ineficiencias; hoy en día pedirán soluciones a sus administraciones en cuanto a las aceras sin espacio para pasar, señalización confusa 0 insuficiente, nuevos equipamientos sin accesos adecuados, mantenimiento de calles poco racional, semáforos poco coordinados que no incluyen a todos los usuarios de la vía pública, redes de suministros independientes, servicios de emergencia que tardan demasiado en llegar, 
transporte colectivo ineficiente y distribución de mercancías caótica; en síntesis, son los ciudadanos quienes demandan soluciones rápidas a la variopinta e infinidad de iniquidades del sistema.

Un planteamiento como el descrito anteriormente, exige a aquellas ciudades que aspiren a ser consideradas como 'Inteligentes' a renovar y mejorar constantemente las soluciones tecnológicas aplicadas con el fin de mejorar la eficiencia de sus procesos y la calidad de vida en su entorno. Se parte entonces de un principio la categoría "Inteligente" no es duradera, es decir, no está asociada a la consecución de una meta, sino que implica más bien el compromiso por parte de los distintos agentes involucrados en un proceso constante de mejora, con un potencial casi infinito, y una meta no menos lejana (Hernández, 2011).

El término "Ciudades Inteligentes" no se utiliza para una ciudad con ciertos atributos, es empleado para definir los diversos aspectos que van desde el concepto de una ciudad inteligente con respecto a la educación de sus habitantes en asociación con la economía incluso con los puestos de trabajos, pues en ellos reside la inteligencia de la industria y los sectores de producción tradicionales como aquellas las industrias que manejan los campos de la información y tecnologías de la comunicación (TIC), así como otras industrias que emplean las TIC en sus procesos de producción (Vienna, 2007).

De igual manera, el término "Ciudades Inteligentes" también es vinculante con la educación y su relación con sus habitantes. Una ciudad inteligente tiene por lo tanto, habitantes inteligentes en términos de educación. Otra retórica de ciudad inteligente, se refiere a la relación entre el gobierno de la ciudad respectivamente. A su administración y la de sus ciudadanos. La buena gobernanza como un aspecto de una buena administración donde la población se es tenida en cuanta valorada y sobre todo donde se logra velar por sus derechos de una manera legal vigente y transparente. A menudo también se refiere a la utilización de nuevos 
canales de comunicación para los ciudadanos, por ejemplo, "Gobernanza electrónica" o "Democracia electrónica".

Ciudad inteligente, es una suma de particularidades que lleva el usufructo de las tecnologías a su entramado social para discutir el uso de la tecnología moderna en la vida urbana cotidiana; transporte, logística, así como nuevos sistemas de transporte "Inteligentes" sistemas que mejoren el tráfico urbano y la movilidad de los habitantes y un sinfín de aspectos que se refieren a la vida en una ciudad inteligente como: no dañar el medio ambiente; utilización de las tecnologías para la gestión (inteligente) con un fin último: Desarrollo Sostenible (Robusté, 2003).

\section{Características de las ciudades inteligentes}

Hablando en términos generales, hay varias características que tienen en común las distintas ciudades, por lo que se puede englobar en tres temáticas principales:

\section{a.) Desarrollo de la infraestructura}

Se enfoca en crear una excelente infraestructura para mejorar la economía, la política, el desarrollo cultural, social y urbano. Busca crear eficientes canales de comunicación donde los servicios empresariales, de vivienda, ocio, estilo de vida y telecomunicaciones estén muy bien conectados: "Merece mención especial la existencia de iniciativas de ciudad inteligente orientadas a la creación de infraestructuras experimentales de investigación para el avance en temáticas asociadas a las tecnologías relacionadas con la Internet del Futuro y sus aplicaciones" (Hernández, 2011). Se emplean altas tecnologías y creatividad pensando en el crecimiento y evolución urbana, orientada hacia la globalización para garantizar el éxito de la ciudad. 


\section{b.) Transporte y movilidad urbana}

En cuanto al modelo de transporte y movilidad urbana, se pueden realizar innumerables prácticas que conlleven a la reducción de contaminantes y que ayuden al ciudadano a sentirse cómodo con una alta calidad de vida y con todas sus necesidades satisfechas (Ecodez, 2010), entre ellas:

-Análisis de los flujos de tráfico, dando prioridad al transporte de emergencias y al transporte público.

- Detección automática de las infracciones del código de circulación y los peligros en las carreteras información mediante señales adecuadas o información online de los accidentes producidos en las vías de circulación a los vehículos próximos.

- Desarrollo de modelos matemáticos y simulaciones para poder comparar distintas vías de circulación y distintos escenarios de transporte y así poder predecir posibles efectos sociales y ambientales.

- Implantación de servicios de información online para los ciudadanos: búsqueda a través de Smartphone, dispositivos móviles, o pantallas fijas: conexiones, tiempos estimados de llegada del transporte público, servicios para compartir bicicletas o vehículos (Observatorio Tecnológico de la Energía, 2011).

\section{Modelos de ciudades inteligentes en el mundo}

Un modelo de ciudad inteligente es una iniciativa que toman los grupos sociales privados o públicos con el fin de cubrir necesidades que se van generando de algún problema, ya sea medioambiental, organizacional, de negocios entre otros. Ayudan a la ciudad a avanzar y con su avance y compromiso permanente llegan a ser ciudades inteligentes (Hernández, 2011). 
Algunos modelos que han sido implementados en ciudades son:

- Eficiencia y gestión energética

- Entornos de negocio y economía del conocimiento

- Transporte y movilidad urbana

- E-gobierno y participación ciudadana

- Medioambiente

- Urbanismo

- Turismo y actividad cultural

- Sanidad y atención personal

Cada uno de estos modelos logra llevar a una ciudad a la categoría de ciudad inteligente donde se piensa en una ciudad comprometida con el entorno cuyos elementos arquitectónicos son de vanguardia y donde las edificaciones están dotadas de las soluciones tecnológicas más actuales con el fin de facilitar la interacción del ciudadano rural a un entorno urbano organizado y cooperar con el ciudadano común ayudarle hacer su vida más fácil.

\section{MÓDULOS DE INFORMACIÓN. CONSIDERACIONES DE DISEÑO: PROPUESTA DE DISEÑO DE LA INTERFAZ GRÀFICA}

Mediante la revisión de literatura se ha podido precisar que los denominados "kiosco interactivo" permiten incubar conductas de reaprendizaje digital, alfabetizar y contruibuir con la construcción de ciudades inteligente; éstos que no son más que un ordenador instalado en una estructura segura y resistente y que deja a la vista tan sólo aquellos elementos que permiten al usuario acceder a la información o servicios para los que ha sido ideado se constituyen en las células digitales que repercuten en la construcción del nuevo tejido. Por ello, los 
analistas prevén en 2010, que el $75 \%$ de las transacciones realizadas en las principales ciudades del mundo serán a través de terminales de autogestión (Plexus, 2010).

Estos se integran además, en las actuales estrategias de acceso multicanal, configurándose, como un canal complementario para el despliegue de servicios electrónicos, contención de costes y mejora del servicio a clientes, empleados y público en general. En la banca, los cajeros automáticos junto con Internet, se han convertido en el punto de contacto más utilizado por los usuarios.

Por otra parte, un módulo está conformado por una pantalla o monitor sensible al tacto (touchscreen), una computadora que contiene un software interactivo, que administra y contiene la información que se quiere compartir. Todo esto protegido por una estructura que permite la visibilidad y que gracias a un conjunto de características ergonómicas, antivandálicas y pensadas en los diferentes factores climáticos y tecnológicos adecuados a la necesidad del cliente hacen de esta herramienta un aliado perfecto (Keyvolution, 2012).

Así pues, los módulos de información están concebidos para un uso público, tanto en espacios abiertos (vía pública, parques, entrada de edificios administrativos,...) como en recintos cerrados (aeropuertos, terminales, estaciones); y son idóneos para un uso privado y multiusuario en lugares en los que el acceso a equipos informáticos tradicionales se ve dificultado (lonjas, almacenes, servicios técnicos, talleres industriales, etc.)

Por lo general, se construyen en materiales muy resistentes, protegidos contra polvo y humedad, y con sistemas antivandálicos y ofrecen una apariencia adaptada al entorno en el que se sitúan (personalizables) y son altamente configurables en cuanto a equipamiento integrado y periféricos (Plexus, 2010). 
Por otra parte Álvarez (2013), plantea que un kiosco interactivo o módulo informativo es una computadora situada en lugar público que permite a los usuarios realizar múltiples acciones. También se utiliza como herramienta de información y marketing para las empresas. En el presente, los quioscos interactivos a menudo tienen pantallas táctiles.

Así mismo, tienen como objetivo presentar una interfaz amistosa y de fácil interacción que facilite su utilización por cualquier tipo de usuario. Pueden ser personalizados para realizar múltiples tareas de modo que pueda colaborar con los trabajadores del servicio e incluso realizar las funciones de éstos. Al permitir un acceso permanente al público, se pretende incrementar la productividad y mejorar los retornos de inversión. Estos se utilizan en muchas aplicaciones y mercados verticales, tales como: bancos, ventas de entradas de espectáculos, correos, hospitales, aeropuertos, estaciones ferroviarias, grandes supermercados.

Considerando las posturas teóricas y una vez realizadas los análisis para el diseño se procedió a la elaboración teórica- gráfica del modulo informativo interactivo para ser empleado en la Biblioteca Pública del Estado Zulia. Por ello, se procede a detallar aspectos del mismo.

\section{a) Criterios de diseño de la interfaz gráfica}

- Estética: El menú principal tiene un aspecto limpio y ordenado y los íconos son fáciles de entender. Se manejó la misma gama de colores contrastantes, haciendo que el menú tenga un aspecto unificado.

Los íconos en general son explícitos, aunque estéticamente agradables y sencillos en color plano sin degradado para una fácil lectura y visualización de los mismos. El menú principal, al igual que los elementos de la interfaz 
presentan un aspecto consistente, en vez de usar una combinación de colores que van de los tierra (marrones) al naranja y el blanco.

- Diversión de la Interacción: la interfaz cuenta con una interacción libre para el usuario, el cual puede ir al lugar que más le interese dentro de la misma, de igual modo esta va referida a desplazamientos de pantalla, acciones de ampliar, pellizcar; se pensó para pantallas táctiles con un sistema de software libre, lo que permite que el usuario esté en presencia de una interfaz fácil de utilizar y entender; y por consiguiente que sea de su agrado, buscando así interacciones intuitivas que permiten el fácil entendimiento y utilización de la misma.

\section{b) Diseño Conceptual de la Interfaz de Usuario}

Originalmente, se determinó que la interfaz gráfica del módulo informativo estaría basada en formas geométricas redondeadas y simplificadas, debido a que

éstas son reconocidas como amigables, fácil de entender y accesibles. Sin embargo, de igual manera, la interfaz se desarrolló con un diseño plano o flat buscando una optimización de los elementos, sencillez y sobre todo un espacio sencillo y amigable que se adapte a las necesidades de los usuarios con capacidades diversas que visitan la Biblioteca Pública del estado Zulia "María Calcaño".

Por otra parte, se empleó la imagen de la fachada de la biblioteca buscando que los usuarios se sientan identificados con la estructura física de las instalaciones, y su apariencia real, además, de buscar dar una sensación de estar dentro de la misma a través del uso de la interfaz en el modulo informativo

\section{c) Diagramación de la Interfaz}

La interfaz de usuario se encuentra diagramada a cuatro columnas buscando que los contenidos se encuentren ordenados, legibles y con facilidad para ser 
encontrados, de igual manera, esto permite una perfecta alineación de los elementos en el espacio para brindar orden visual y armonía de los contenidos con los elementos gráficos. El aspecto de la interfaz es atractivo y limpio, con pocos elementos para permitir que el usuario pueda concentrarse en el contenido.

\section{d) Colores empleados}

Los colores empleados fueron: cálidos, neutros y contrastantes buscando alto contraste entre los elementos, e fondo que es de color blanco y las imágenes, los mismos se encuentran empleados de la siguiente manera:

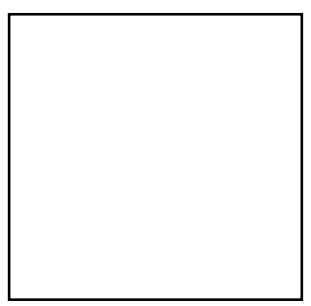

Color blanco: para el fondo de la interfaz, buscando mayor contraste y simplicidad en el diseño y con los elementos, así mismo se emplea como fondo a las opciones seleccionadas en el menú principal para que el usuario sepa en qué sección se encuentra posicionado

Color Naranja: color cálido empleado para la barra de la

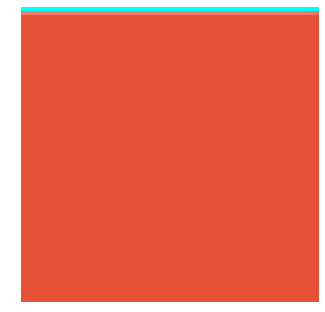
cabecera y el primer fondo de los textos de la interfaz, al igual que para los botones de leer más o ampliar los contenidos
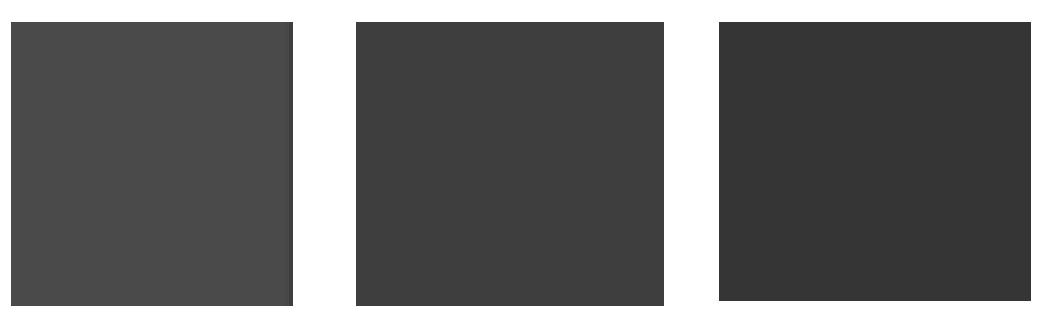

Degradaciones del color gris del más claro al más oscuro para los fondos de las 3 columnas restantes de los textos, buscando contraste con el color naranja al igual que con el blanco de los textos para mayor legibilidad, así mismo, el gris será el color empleado en los iconos y elementos que se utilizaran sobre el fondo blanco. 
De igual manera, se emplearon colores tierra para la representación de la fachada de la Biblioteca Pública María Calcaño.

\section{e) Tipografía}

La tipografía fue Candara en forma negrita, en la barra del menú se encuentra en un tamaño de 18 puntos mayúscula y en color blanco para lograr gran contraste con la barra naranja sobre la que se dispone.

De igual menara, para los títulos la tipografía es de 30 puntos mayúscula y en color gris la primera palabra y naranja las que siguen ya que se encuentran sobre fondo blanco buscando mayor contraste para una mejor legibilidad y visualización de los elementos de la interfaz, a su vez, los textos internos son de color blanco tipo oración a punto 16 igual que los subtítulos que a diferencia de los anteriores se emplean en mayúsculas.

\section{f) Iconos}

El empleo de un grafismo basado en iconos permite que el usuario con rapidez reconozca los elementos a los cuales se hacen referencia de forma escrita lo cual ayuda a que este se posicione de manera más rápida y concisa en la información de su interés. Dichos iconos, se encuentran en positivos y negativos dentro de la interfaz permitiendo crear contraste con los elementos internos y con el fondo permitiendo mejor visualización por parte de los usuarios a los que va destinado el diseño de la interfaz.

\section{g) Interacción}

La interacción es intuitiva y permite al usuario un contacto directo y sencillo, la misma permite al tocar una opción en la pantalla táctil que el usuario avance o retroceda según sea su interés. De esta manera, el usuario tendrá la facilidad de desplazarse en la interfaz según sus intereses, necesidades y gustos, así mimo desplazando sobre la pantalla hacia la derecha o a la izquierda ir adelante o hacia 
atrás al igual que permite la opción de volver con una flecha contenida debajo del icono principal de la sección.

En cuanto a la interacción con los elementos, se constata:

- Experticia inmediata: La interfaz hace uso de las habilidades existentes como el tocar para seleccionar, el arrastrar para mover un elemento y el pellizcar/expandir dos dedos para acercar/alejar una imagen. Los usuarios familiares con estos gestos se convierten en expertos instantáneos.

- Aprendizaje progresivo: Para los usuarios que no son familiares con los gestos mencionados previamente, se muestran ayudas animadas en un orden que permite al usuario iniciar con la tarea básica (mover las pantallas) y progresar hasta la tarea avanzada (acercar/alejar los elementos de la pantalla).

- Interacción directa: Las interacciones son directas, rápidas y con retroalimentación instantánea.

- Carga cognitiva: Las interacciones usadas son pocas y se repiten en todo el sistema, por lo que se mantiene una baja carga cognitiva y así crea una experiencia fácil de usar y de aprender.

\section{DISEÑO CONCEPTUAL MODULO INFORMATIVO}

El diseño conceptual del modulo, está basado en formas circulares y rectangulares; aunque no completamente recto sino que cuenta con bordes redondeados y una forma irregular para dar un aspecto más atractivo y así destacarse de su entorno.

Como parte del Diseño, la pantalla muestra el menú principal en vez de presentar un video o publicidad debido a que generalmente al ver una pantalla que 
pasa imágenes las personas asumen que es una pantalla publicitaria solamente y no le prestan más atención. Por esta razón, sobre el mismo sistema se imprimen las palabras "Pantalla Táctil" para que su capacidad táctil se conozca inmediatamente. Además, al ver el menú principal en la pantalla, el usuario puede enterarse rápidamente sobre las funciones que le ofrece el sistema.

Sin embargo, al tener una pantalla estática, el sistema se hace completamente dependiente de su diseño físico para captar la atención del usuario. Por consiguiente, para evitar que el diseño sea el único factor atrayente, se adicionó una banda de LEDs (Light Emitting Diodes) en los laterales del sistema que oscilaría entre los colores propios de la identidad del la Biblioteca Publica María Calcaño. Este cambio de colores sería suave para no ser irritante pero también lo suficientemente notable para resaltar entre el entorno y así captar la atención de cualquier individuo que se encuentre en el área.

Por otra parte, el módulo de información está pensado para ser utilizado por los usuarios de la Biblioteca con especial atención a los usuarios con cualquier tipo de discapacidad, ya que mismo contara con un lector óptico que facilitara el acceso a personas con discapacidad motora total, de igual manera lector de pantalla con audio para la personas no videntes y el recorrido de lectura para las personas con discapacidad auditiva permitiendo la inclusión e integración total con la información en los espacios bibliotecarios.

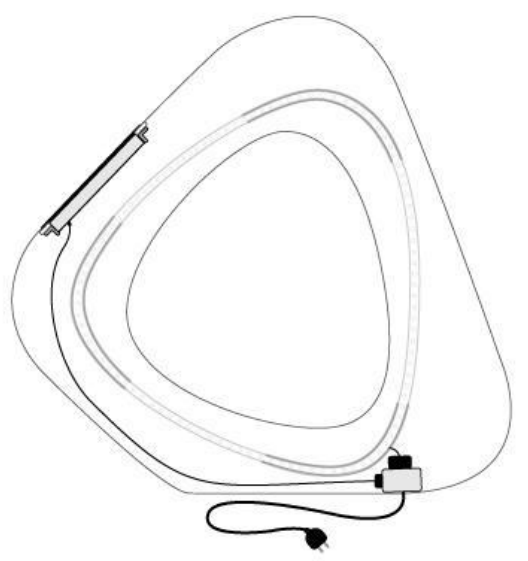

Figura 1. Forma del módulo. 


\section{DIMENSIONES Y ERGONOMÍA}

Las dimensiones presentadas fueron pensadas para la conveniencia de un público amplio, tomando en cuenta las mediciones de los usuarios objetivos, teniendo en cuenta la estatura promedio de los usuarios que es de $1.75 \mathrm{~m}$ para los hombres y $1.60 \mathrm{~m}$ para las mujeres. Por esta razón, la pantalla se posicionó a una altura relativamente baja y con una inclinación de 45 grados, un ángulo cómodo para las pantallas táctiles y para la lectura en general. Esto permite que la pantalla sea alcanzable y cómoda de usar para personas de estaturas altas, medianas y bajas, así como para personas con discapacidad motora.

La pantalla es de 24 pulgadas $(53 \mathrm{~cm} \times 30 \mathrm{~cm}$ ) y el ancho del módulo es de $73 \mathrm{~cm}$, dejando un espacio amplio para la interacción y permitiendo que los códigos QR mostrados sean lo suficientemente grandes para su fácil escaneo.

En la parte posterior del módulo, hay un espacio para un cartel A1 $(59.4 \mathrm{~cm} x$ $84.1 \mathrm{~cm}$ ) y cuenta con una inclinación de 30 grados para permitir la lectura cómoda de la información que se encuentre en el cartel.

Se consideró implementar un sistema de ajuste automático para permitir que la altura de la pantalla fuera graduable; y así brindar mayor conveniencia a todo tipo de usuario. Ver Figura 2.

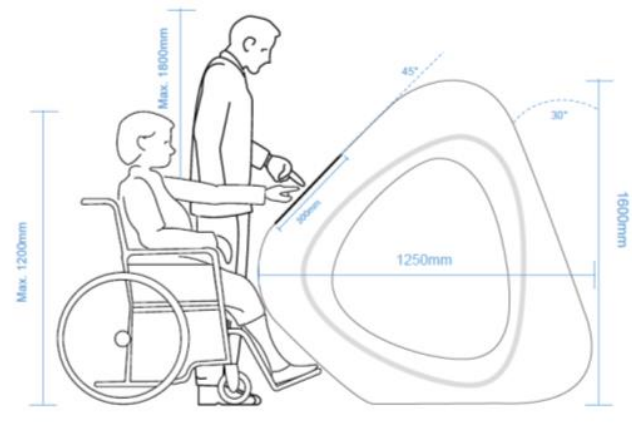

Figura 2. Medidas del módulo de información con respecto al canon humano 


\section{REFLEXIONES FINALES}

Una vez efectuados los estudios en torno a la relación ciudades inteligentes - módulos de información se obtiene que los módulos recrean y permean micro entornos inteligentes que contribuyen a la articulación de las redes y el desarrollo de macro entornos sustentables para el fomento de Ciudades Inteligentes. De esta forma, los módulos son herramientas de aprendizaje e inclusión para el sector comercial y turístico los módulos también son de gran utilidad, al brindar información oportuna sobre los diferentes servicios y negocios que conforman un sector; sirven además como herramienta educativa y guía turística divulgan información pedagógica en puntos de interés cultural, histórico y recreativo; y finalmente el módulo interactivo puede mostrar y clasificar su información mediante mapas, listas, sectores de usuarios y muchas más; son en efecto, empleados en una variedad de áreas como la educación, el entretenimiento, la medicina y la publicidad por su capacidad de convertir al espectador en un usuario interactivo (Car, 1998).

Gracias al uso de un sistema interactivo los usuarios desarrollan un mejor proceso de adaptación y usos de las tecnologías, el cual radica en el uso accesible de la información que permiten un acercamiento intimo entre el ciudadano con su acontecer al igual que con el conocimiento. En este sentido, dichos sistemas permiten una interacción íntima con el ordenador y las diversas tecnologías volviéndose de esta manera en sistemas más amigables, intentando así, generar inclusión en la diversidad, aprendizaje y por supuesto el uso de las tecnologías para la gestión (inteligente) cuyo fin último debe ser el desarrollo sostenible del entorno y minimizar las brechas existentes.

Se asume además, que son las bibliotecas los centros de reentrenamiento digital y espacios multiplicadores de la inteligencia social capaces de fomentar el concepto emergente de Ciudad Inteligente; razón por la cual, y desde esta perspectiva, el uso de las llamadas tecnologías de la información y de la comunicación (TIC) aplicadas en el funcionamiento de la biblioteca y el impacto 
causado por este uso de las TIC, son factores conducentes para generar una nueva forma de la accesibilidad en la vida económica, social, cultural y política de los ciudadanos.

Se insiste que, las bibliotecas son espacios fundamentales de acceso a la cultura, la formación, aprendizaje, gestión del conocimiento, inclusión y el disfrute intelectual; suponen además un bien incuestionable para las personas y en especial para las personas con capacidades diversas, y a su vez, una herramienta indispensable para su desarrollo intelectual.

Se concluye que, gracias al empleo de un diseño minimalista de los módulos, pero atractivo y adaptado a las necesidades físicas de los usuarios - espacio de interacción se fomenta el aprendizaje digital, la inclusión y el desarrollo de las denominadas Ciudades Inteligentes.

\section{REFERENCIAS DOCUMENTALES}

AGUIRRE, Eduardo (2013). "URBOTICA Y CIUDADES INTELIGENTES". Articulo consultado en www.lavoz.ar

BALBO, Marcello (2003). "La ciudad Inclusiva". Publicación de las Naciones Unidas, Santiago de Chile. 2003.

BERMÚDEZ, Pablo (2012). LIBRO BLANCO SOBRE CIUDADES DIGITALES EN IBEROAMÉRICA. Asociación Hispanoamericana de Centros de Investigación y Empresas de Telecomunicación.

BONILLA, Martha (2012). El futuro de las ciudades: Información e inclusión. Foro "Ciudades Inteligentes". 
DI SIENA, Domenico (2009). "ESPACIOS SENSIBLES Hibridación físico-digital para la revitalización de los espacios públicos". Programa de Doctorado Periferias, sostenibilidad y vitalidad urbana del Departamento de Urbanística y Ordenación del Territorio. Madrid, España.

GONZÁLEZ, Vicente (2008). "Ciudades inteligentes en la planificación contemporánea". Jornadas Ciudad e Innovación. Valencia, España 2008.

HENRIQUEZ, Milagros (2012). "INCLUSIÓN O INTEGRACIÓN DE LAS PERSONAS CON DISCAPACIDAD"

HUERTA, Jaime (2007), "DISCAPACIDAD Y DISEÑO ACCESIBLE". Diseño urbano y arquitectónico para personas con discapacidad. Lima, Perú.

MANUAL DE ACCESIBILIDAD. Comisión nacional asesora para la integración de personas discapacitadas. Consejo nacional de coordinación de políticas sociales presidencia de la nación. Venezuela 2008.

MÉNDEZ, Mariela (2013). "DISEÑO PARA LA CREACIÓN DE KIOSCOS TURÍSTICOS EN LAS AVENIDAS GUAYAQUIL Y 9 DE OCTUBRE POR MEDIO DEL MUNICIPIO, PARA PROMOVER EL TURISMO EN EL CANTÓN NARANJITO". Universidad Estatal de Milagro Unidad Académica de Ciencias Administrativas y Comerciales en Ecuador.

MITCHELL, William (2007). "Ciudades inteligentes”. LECCIÓN INAUGURAL DEL CURSO 2007-2008 DE LA UOC. Octubre de 2007.

OBSERVATORIO TECNOLOGICO de la ENERGIA, (2011). "Mapa tecnológico, ciudades inteligentes" 
ORTIZ, Claudia (2008). "LA ACCESIBILIDAD ESPACIAL EN LA DEFINICIÓN DE TERRITORIOS INTELIGENTES". Caracas, Venezuela.

RAMOS Erick, (2003). "USO DE LAS TECNOLOGÍAS DE INFORMACIÓN EN PERSONAS CON DISCAPACIDADES", Universidad Rafael Belloso Chacín (URBE).

SÁNCHEZ B. y Vargas M. (2006), "BIBLIOTECAS VIRTUALES ADAPTABLES: UN DESAFÍO DE LA SOCIEDAD DE LA INFORMACIÓN". La Habana

VALENCIA López y Cárdenas Restrepo (2004)." ANÁLISIS Y DISEÑO DE UN MODELO INFORMÁTICO PARA UNA BIBLIOTECA DIGITAL UTILIZANDO AGENTES INTELIGENTES EN LA GESTIÓN Y CONSULTA DE INFORMACIÓN". Universidad Tecnológica de Pereira, Facultad de Ciencias Básicas, Colombia.

VÁZQUEZ, (2012). "CIUDADES INTELIGENTES Y MODELOS LOGÍSTICOS DE CIUDAD", Universidad de Buenaventura seccional Medellín. 